\title{
Modelling of Spin-Dependent Mechanical Friction at Atomic Level
}

\author{
M. Gzik-Szumiata ${ }^{a, *}$, T. Szumiata ${ }^{a}$ And D. Morozow ${ }^{b}$ \\ ${ }^{a}$ Department of Physics, University of Technology and Humanities in Radom, Krasickiego 54, 26-600 Radom, Poland \\ ${ }^{b}$ Institute of Machinery Engineering, University of Technology and Humanities in Radom, \\ Krasickiego 54, 26-600 Radom, Poland
}

\begin{abstract}
In this work a simple 2D model of pseudostatic friction at atomic level has been prepared, in the frame of which both Lennard-Jones potential and spin-dependent term of exchange interaction has been included. As an example, it has been demonstrated, that for iron both average lateral and normal forces between atoms of "base" and "slider" in the tribological node are altered through the change of relative direction of spins, by over a dozen of percent, when the interatomic distance is comparable to the lattice constant. Spin-dependent correction of atomic-level friction coefficient has been estimated.
\end{abstract}

DOI: 10.12693/APhysPolA.133.713

PACS/topics: 62.20.Qp, 46.55.+d, 75.30.Et, 71.70.Gm, 75.50.Bb, 75.45.+j

\section{Introduction}

Though magnetic coupling is considered to be noticeably weaker than chemical, electronic interactions, it has been recently shown by Wolter and co-workers [1] that spin-spin exchange energy can considerably influence the friction between magnetic materials. This achievement has been enthusiastically commented in $[2,3]$, though the work [1] presents not purely experimental results but combination of spin-polarized scanning tunnelling microscopy (SP-STM) outcomes and theoretical reconstruction of frictional phenomena by means of MonteCarlo simulations. The results reported in [1] have been qualitatively confirmed in very recent work [4] based on $a b$ initio, first-principles calculations in the frame of density functional theory (DFT).

The aim of present work was to prepare possibly simple model which could predict spin-dependent phenomena of friction for different magnetic systems. According to the review articles $[5,6]$, it is still reasonable to develop semianalytical, basic models of friction [7, 8] (like PrandtlTomlinson and Frenkel-Kontorova ones), which are more universal and less time-consuming than more advanced molecular dynamics (MD) simulations [9].

\section{Theoretical model}

The basic interatomic interactions have been described by standard Lennard-Jones (L-J) potential:

$$
U_{L J}\left(r_{i j}\right)=4 \varepsilon_{i j}\left[\left(\frac{\sigma_{i j}}{r_{i j}}\right)^{12}-\left(\frac{\sigma_{i j}}{r_{i j}}\right)^{6}\right]
$$

where $r_{i j}$ is a distance between atoms $i$ and $j, \varepsilon_{i j}$ - depth of the potential ("bonding energy"), $\sqrt[6]{2} \sigma_{i j}$ - the distance

\footnotetext{
*corresponding author; e-mail: m.gzik@uthrad.pl
}

corresponding to minimum of the potential ("length of the bond"). Additionally, the spin-dependent part of the interactions has been introduced in the form of Heisenberg exchange energy:

$$
U_{e x}\left(r_{i j}\right)=-2 J_{i j} \mathrm{e}^{-\frac{r_{i j}-r_{N N}}{\lambda_{i j}}} \boldsymbol{S}_{i} \circ \boldsymbol{S}_{j}
$$

where, $\boldsymbol{S}_{i}$ and $\boldsymbol{S}_{j}$ are spins of atoms $\left(\left|\boldsymbol{S}_{i}\right|=\left|\boldsymbol{S}_{i}\right|=1\right)$, $J_{i j}$ - exchange integral for the distance of the nearest neighbours $r_{N N}$. An exponential drop of the exchange interaction energy with interatomic distance has been assumed with $\lambda_{i j}$ "decay constant". The interatomic forces in $2 \mathrm{D}$ geometry can be expressed as follows:

$$
\begin{aligned}
& F_{x}\left(x_{i j}, y_{i j}\right)=-\frac{\mathrm{d}}{\mathrm{d} x_{i j}}\left[U_{L J}\left(r_{i j}\right)+U_{e x}\left(r_{i j}\right)\right] \\
& F_{y}\left(x_{i j}, y_{i j}\right)=-\frac{\mathrm{d}}{\mathrm{d} y_{i j}}\left[U_{L J}\left(r_{i j}\right)+U_{e x}\left(r_{i j}\right)\right]
\end{aligned}
$$

where $r_{i j}=\sqrt{x_{i j}^{2}+y_{i j}^{2}}$.

The simple system of atoms has been considered within model. The "slider" in frictional pair is represented by atom (1) gradually dragged above the "base" at height $h$. The "base" is constituted with atom (2) elastically bonded to three rigidly constrained neighbors (3), (4), (5) situated respectively beneath, left-side and rightside at the distance of "lattice constant" $a_{0}$ (equal to $r_{N N}=\sqrt[6]{2} \sigma_{i j}$ ). Quasi-static equilibrium conditions for atom (2) are following:

$$
\begin{aligned}
& F_{x}\left(x_{2}-x_{1}, y_{2}-y_{1}\right)+F_{x}\left(x_{2}-x_{3}, y_{2}-y_{3}\right) \\
& \quad+F_{x}\left(x_{2}-x_{4}, y_{2}-y_{4}\right)+F_{x}\left(x_{2}-x_{5}, y_{2}-y_{5}\right)=0, \\
& F_{y}\left(x_{2}-x_{1}, y_{2}-y_{1}\right)+F_{y}\left(x_{2}-x_{3}, y_{2}-y_{3}\right) \\
& \quad+F_{y}\left(x_{2}-x_{4}, y_{2}-y_{4}\right)+F_{y}\left(x_{2}-x_{5}, y_{2}-y_{5}\right)=0 .
\end{aligned}
$$

After numerical solving of this system of nonlinear algebraic equations with Levenberg-Marquardt method implemented in PTC Mathcad software one obtains the de- 
pendencies of the coordinates $\left[X_{2}\left(x_{1}\right), Y_{2}\left(x_{1}\right)\right]$ of atom (2) on the $x_{1}$ position of "slider" atom (1). This enables to express local values both of lateral $(x)$ and normal $(y)$ force acting on atom (1) as a function of $x_{1}$ :

$$
\begin{aligned}
& F_{x}\left(x_{1}-X_{2}\left(x_{1}\right), y_{1}-Y_{2}\left(x_{1}\right)\right), \\
& F_{y}\left(x_{1}-X_{2}\left(x_{1}\right), y_{1}-Y_{2}\left(x_{1}\right)\right) .
\end{aligned}
$$

The mean values of lateral and normal force are defined as follows:

$$
\begin{aligned}
& \left\langle F_{x}\right\rangle=\frac{1}{a_{0}} \int_{-a_{0}}^{0} \llbracket F_{x}\left(x_{1}-X_{2}\left(x_{1}\right), y_{1}-Y_{2}\left(x_{1}\right)\right) \rrbracket \mathrm{d} x_{1} \\
& \left\langle F_{y}\right\rangle=\frac{1}{a_{0}} \int_{-a_{0}}^{0}\left\|F_{y}\left(x_{1}-X_{2}\left(x_{1}\right), y_{1}-Y_{2}\left(x_{1}\right)\right)\right\| \mathrm{d} x_{1}
\end{aligned}
$$

where operator $\llbracket \cdot \rrbracket$ chooses only the ranges with negative $F_{x}$ and return its module, whereas the operator $\|\cdot\|$ chooses only the ranges with positive $F_{y}$. Such definitions consider only the regions of repulsive interactions and non-equilibrium character of the process, when after exceeding a critical point $x_{1}=0$ the "slider" atom (1) jumps to another potential well dissipating the energy via transfer into the crystalline lattice degrees of freedom. The calculated mean values of lateral and normal forces allow to find atomic friction coefficient:

$$
\mu=\frac{\left\langle F_{x}\right\rangle}{\left\langle F_{y}\right\rangle} .
$$

\section{Results and discussion}

The numerical calculations have been performed for the case of iron atoms. The values of L-J potential parameters and have been estimated considering the nearest neighbors distance and cohesive energy corresponding to real Fe monocrystal of bcc structure [10]. Recent spin-polarized electronic structure calculations predict the value of the effective exchange integral for bcc iron greater than $0.01 \mathrm{eV}$ [11]. Moreover, it has been assumed, that "decay constant" is equal to the distance for bcc iron.

In Fig. 1 the dependence of mean lateral and normal forces $\left(\left\langle F_{x 00}\right\rangle\right.$ and $\left.\left\langle F_{y 00}\right\rangle\right)$ as a function of the distance $h$ between "slider" atom and base surface is presented for the case without spin-spin interactions. Analogous predictions for atomic friction coefficient are displayed in Fig. 2. The strong increase of friction intensity is clearly seen when diminishing the distance $h$ well below one lattice constant.

After "switching on" the exchange interactions the difference both of lateral and normal force occurs for antiparallel $(\uparrow \uparrow)$ and parallel $(\uparrow \downarrow)$ configurations of spin in "slider" and "base" atoms (Fig. 3). It results in spin-dependent atomic friction coefficient. The relative value of its difference for antiparallel and parallel cases $\left(\mu_{\uparrow \downarrow}-\mu_{\uparrow \uparrow}\right) / \mu_{00}$ becomes important for $h$ values close to one lattice constant, i.e. in the case of weak "electronic friction" (Fig. 4). It reaches dozen percent for $h=0.99 a_{0}$, when $J=0.01 \mathrm{eV}$. For $J=0.05 \mathrm{eV}$ it happens, when $h=0.95 a_{0}$. This case of greater $J$ value in iron (or steel) could correspond to the effect of exchange interactions enhancement caused by the presence of defects, vacancies or impurities [12] the concentration of which is expected to be significant at the surface of frictional pair. It could be a reason of local modifications of spindependent contribution to the friction at atomic level.

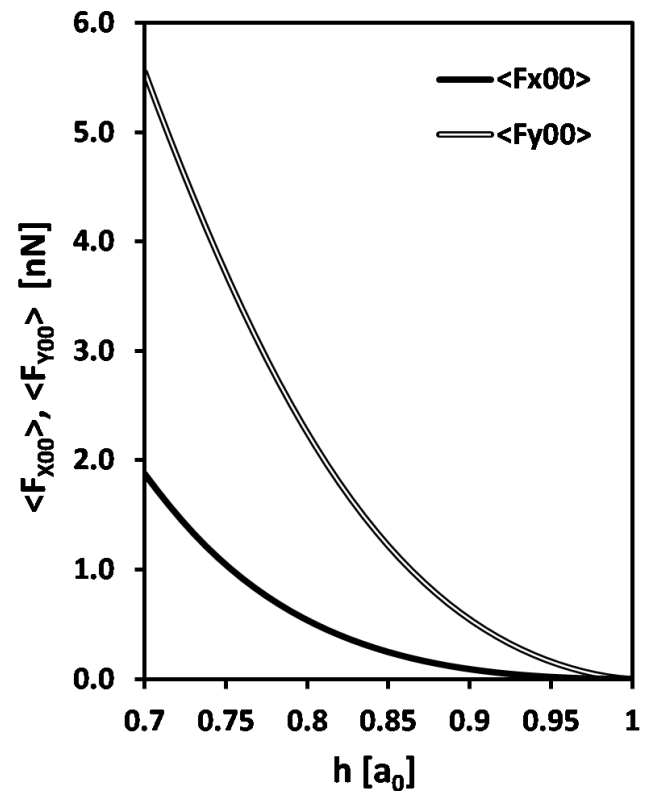

Fig. 1. Dependence of mean lateral $(x)$ and normal $(y)$ force as a function of the distance between slider atom and base surface in the case of no spin-spin interactions.

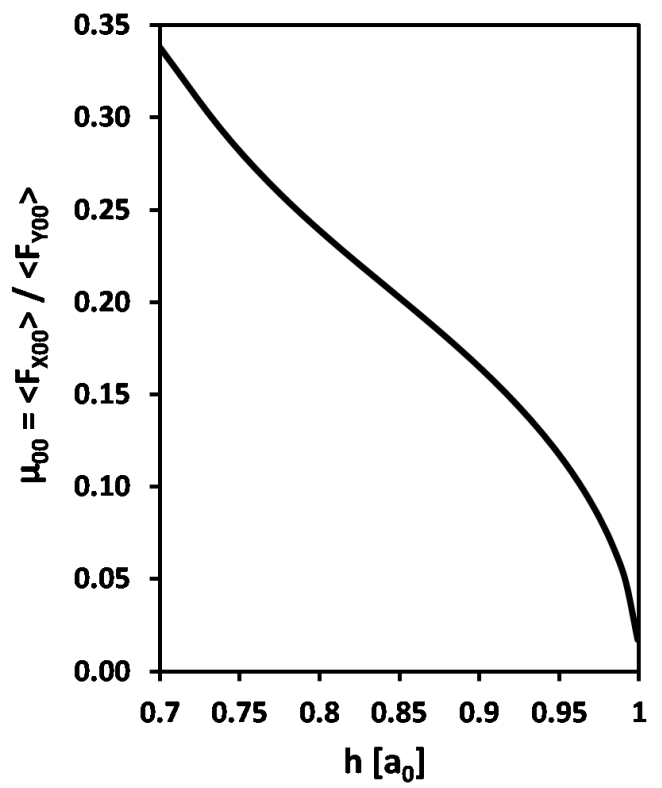

Fig. 2. Dependence of mean atomic friction coefficient as a function of the distance between slider atom and base surface in the case of no spin-spin interactions. 


\section{Conclusions}

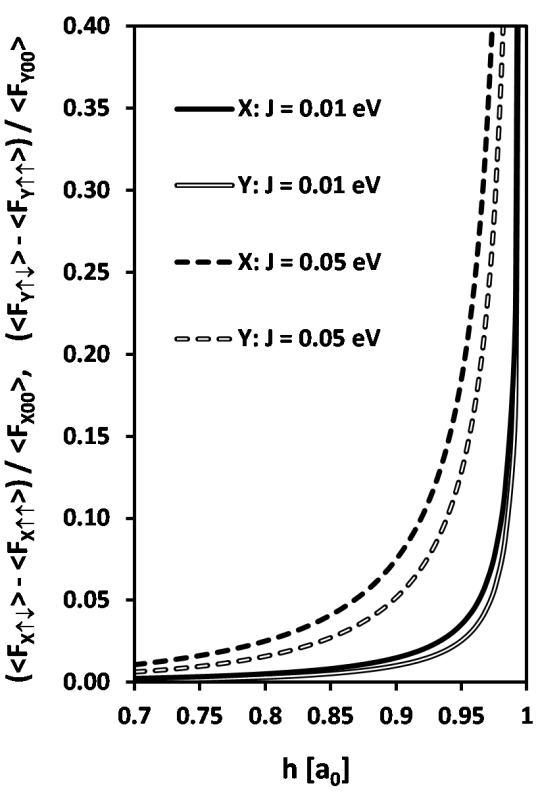

Fig. 3. Dependence of reduced difference both of mean lateral $(x)$ and normal $(y)$ force for antiparallel and parallel spin configurations as a function of the distance between slider atom and base surface. Results for two different values of exchange integral $J$ are presented.

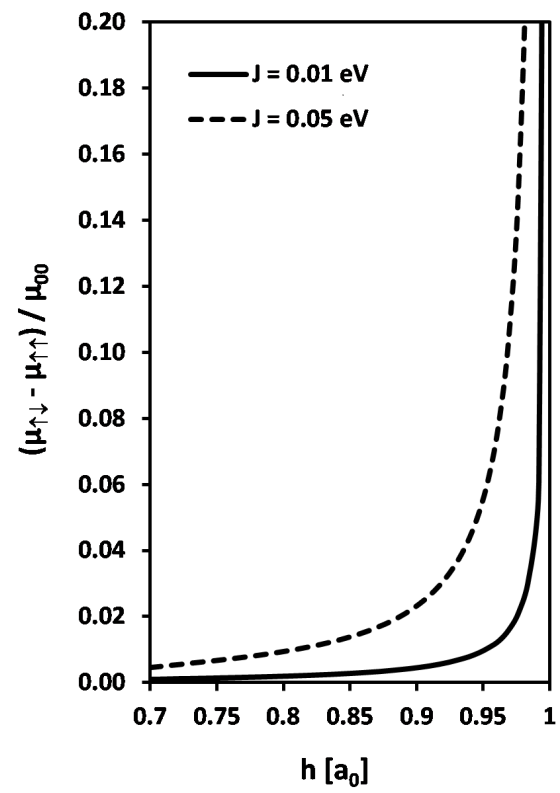

Fig. 4. Dependence of reduced difference both of mean atomic friction coefficient for antiparallel and parallel spin configurations as a function of the distance between slider atom and base surface. Results for two different values of exchange integral $J$ are presented.
The developed model effectively predicts the influence of the exchange interactions of the friction phenomena at atomic level. The estimated order of magnitude of spin-dependent friction contribution coincides with pioneer outcomes of combined experimental and theoretical studies concerning Mn/W(110) thin film [1,4]. Moreover, a big advantage of the model is its simplicity, which enables quick numerical calculations for different frictional pairs. The important challenge for future is the extension of present model from the pseudostatic approach to the dynamic one in order to find the dependence of spin friction on sliding velocity. Another questions would be the role of dipolar magnetic interactions (already investigated in [13] but not together with "electronic friction") as well as the influence of magnetic field on the friction process via magnetoelastic phenomena in nano-scale.

\section{Acknowledgement}

This work was supported by the University of Technology and Humanities in Radom under statutory grant $3179 / 25 / \mathrm{P}$.

\section{References}

[1] B. Wolter, Y. Yoshida, A. Kubetzka, S.-W. Hla, K. Von Bergmann, R. Wiesendanger, Phys. Rev. Lett. 109, 116102-1 (2012).

[2] A. Heinrich, Physics (American Physical Society Ed.) 5, 102 (2012).

[3] T. Commissariat, IOP Physics World, News Sept. 26 (2012).

[4] X. Cai, J. Wang, J. Li, Q. Sun, Y. Jia, Trib. Int. 95, 419 (2016).

[5] J. Nian, Y. Si, Z. Guo, Trib. Int. 94, 1 (2016).

[6] M. Urbakh, E. Meyer, Nature Mater. 9, 8 (2010).

[7] H. Hölscher, A. Schirmeisen, U.D. Schwarz, Phil. Trans. R. Soc. A 366, 1383 (2008).

[8] D. Tománek, Springer Series in Surface Sciences 29 , 269 (1993).

[9] L.C. Zhang, K.L. Johnson, W.C.D. Cheong, Trib. Lett. 10, 23 (2001).

[10] X. D. Dai, Y. Kong, J. Li, and B. X. Liu, J. Phys.: Condens. Matter 18, 4527 (2006).

[11] H. Wang, P.-W. Ma, C.H. Woo, Phys. Rev. B 82 , 144304 (2010).

[12] D. Böttcher, A. Ernst, J. Henk, J. Magn. Magn. Mater. 324, 610 (2012).

[13] C. Fusco, D.E. Wolf, U. Nowak, Phys. Rev. B 77, 174426 (2008). 